\title{
AVALIAÇÃO DE FERRAMENTAS DE COMUNICAÇÃO A DISTÂNCIA NAS ATIVIDADES DE MONITORIA PARTICIPATIVA NO ENSINO DE ENGENHARIA.
}

\author{
DOI: 10.37702/2175-957X.COBENGE.2021.3515
}

Mário Sérgio Oliveira César Filho - ms.filho@hotmail.com

Universidade Federal do Ceará

Rua Professor José Artur de Carvalho 2817

60831-370 - Fortaleza - CE

Leonardo Melo Bezerra - leonardo.bezerra@ufc.br

Universidade Federal do Ceará

Campus Universitário do Pici, Bloco 733733

60455-760 - Fortaleza - CE

Alexandre Miranda Mont'Alverne - montalverne@ufc.br

Universidade Federal do Ceará

Rua João Cordeiro 1095

60110-301 - Fortaleza - CE

Resumo: Os cursos de engenharia, que transcorriam no formato presencial, viram-se diante de uma nova realidade em 2020. Com a pandemia do novo Coronavírus, todos os cursos foram forçados a migrar, emergencialmente, para o formato a distância, com isso, a metodologia de ensino, antes no formato presencial, teve que ser adaptada. A utilização de ferramentas de comunicação "on-line" foi o primeiro desafio imposto tanto a discentes quanto a docentes, e a busca por um melhor entendimento e utilização eficiente dessas ferramentas na construção do conhecimento dos alunos de engenharia através das atividades de monitoria é o motivo deste trabalho. Sabendo que no ensino remoto, a interação entre os participantes é menos intensa, a metodologia ativa baseada na aprendizagem por problematização, com o auxilio de ferramentas de comunicação, foi experimentada na tentativa de manter o aluno desejoso de construir o seu conhecimento. Observou-se que os estudantes demonstraram satisfação pela comunicação rápida e informal fornecida pelos aplicativos de comunicação. Mesmo com o conteúdo sendo apresentado pelo professor antes da distribuição de atividades aos alunos, e com o monitor disponível a quase qualquer momento via 
aplicativo, alguns alunos ainda sentiram dificuldades no desenvolvimento individual do que foi proposto. Houve a sugestão da produção de vídeos explicativos e de um maior uso de algoritmos computacionais na solução de problemas. Por fim, com a consolidação da disciplina, percebeu-se que as notas dos alunos com maior participação foram significativamente superiores às do restante da turma, demonstrando um resultado positivo da aplicação da metodologia proposta.

Palavras-chave: Monitoria. Ensino de engenharia. Ferramentas de ensino a distância. Pandemia. 


\section{AVALIAÇÃO DE FERRAMENTAS DE COMUNICAÇÃO A DISTÂNCIA NAS ATIVIDADES DE MONITORIA PARTICIPATIVA NO ENSINO DE ENGENHARIA.}

\section{INTRODUÇÃO.}

Com a pandemia do Coronavírus, o ano de 2020 foi marcado pela necessidade de uma reformulação no modo de viver, necessitando do distanciamento social, o que levou à suspensão das aulas presenciais, surgindo então a necessidade da realização do ensino remoto não somente nas universidades, mas também no ensino fundamental e médio. A Educação a Distância (EAD), como já diz o próprio nome, trata-se de educar/ensinar remotamente, abandonando a tradicional sala de aula como ambiente de ensino e integrando-o a plataformas digitais na qual o aluno tem acesso a qualquer hora e qualquer lugar. Moran (2011) retrata que muitas instituições banalizam essa forma de ensino, ressaltando a dificuldade de professores e alunos na adaptação à EAD, e alegando previsibilidade de muitos cursos com informações simplificadas, conteúdo raso e poucas atividades estimulantes em ambientes pobres.

Segundo DIESEL, BALDEZ E MARTINS (2017), a utilização de novos recursos tecnológicos durante as aulas não altera o cenário de insatisfação coletiva, marcado por uma dualidade residente nos discursos comumente verbalizados por docentes e estudantes em que estes últimos reclamam das aulas rotineiras, enfadonhas e pouco dinâmicas, ao passo que os primeiros destacam a frustração pela pouca participação, desinteresse e desvalorização por parte dos estudantes em relação às aulas e às estratégias criadas para chamar a atenção destes. Portanto, a tecnologia sozinha não garante aprendizagem.

Embora as disciplinas de mecânica se baseiem em conceitos físicos e matemáticos um tanto quanto triviais, as variações geométricas e espaciais das inúmeras condições de análise maximizam a necessidade de aperfeiçoamento do aluno, o qual pode ser conseguido pela prática intensiva. BARBOSA E MOURA (2014) ressaltam que o ensino em Engenharia deve favorecer o uso dos recursos da inteligência, gerando habilidades em resolver problemas. Nesse caso a escolha de uma metodologia ativa associada à EAD é uma alternativa para a aprendizagem do discente, na tentativa de assim contornar os problemas de didática estereotipados do ensino remoto.

A Metodologia Ativa é uma forma de ensino que muda o papel do aluno em sala de aula, fazendo com que ele seja incentivado a abandonar a postura passiva de apenas observar e escutar o professor, passando a participar ativamente da aula, seja na discussão de um tema ou na resolução de exercícios. Dessa forma, o aluno passa a ser o foco da atividade, sendo ele mesmo responsável pela construção do conhecimento.

De acordo com MACEDO ET AL (2018) a Metodologia Ativa (MA) tem uma concepção de educação crítico-reflexiva com base no processo ensino-aprendizado, resultando em envolvimento por parte do educado na busca por conhecimento. Dentro do conceito de metodologia ativa, existe o método a partir da construção de uma situação problema (SP), a qual proporciona uma reflexão crítica; mobiliza o educando a buscar o conhecimento, a fim de solucionar a SP; ajuda na reflexão e a proposição de soluções mais adequadas e corretas. As concepções teóricas e metodológicas da MA convergem com a Metodologia da Problematização (MP).

Segundo CASTRO, GONÇALVES E BESSA (2017) a metodologia de problematização com o arco de Maguerez pressupõe um aluno ativo, protagonista no processo de construção do conhecimento. O professor assume um papel de orientador, um papel importante na construção metodológica do processo, e não mais como fonte central 
de informação ou de decisão das ações como nos modelos anteriores. Dessa forma os estudantes observam uma parcela da realidade, define um problema de estudo até a realização de algum grau de intervenção naquela parcela da realidade, a fim de contribuir para a sua transformação.

Essa metodologia, para que permita o desenvolvimento dos alunos, requer intenso acompanhamento do docente ou do monitor, o que faz necessária a busca por meios mais eficazes de comunicação com a turma:

- $\quad$ Sendo o WhatsApp o principal aplicativo de comunicação rápida utilizado no Brasil, o seu uso possibilitando a criação de um grupo torna-se quase indispensável;

- $\quad$ A organização do material de acompanhamento foi feita no Google Drive, que é bem simples de ser trabalhado nos quesitos de acessibilidade. Nele constam listas de exercícios aplicadas ao longo da disciplina, resolução dessas questões pelo monitor e bibliografia complementar, como textos e artigos referentes ao assunto abordado;

- A partir desse material, foi atribuída aos alunos uma quantidade de exercícios a serem entregues semanalmente. ARAÚJO (2009) critica o processo formal de avaliação dos alunos, em que os professores tendem a valorizar mais o resultado do que a construção da solução. Dessa forma, a avaliação do trabalho foi catalogada como Satisfatório (S), Insatisfatório (I) ou Falta (F). A caráter de estímulo, o professor sugeriu atribuir a esses trabalhos uma pontuação extra à nota final, sendo a pontuação a média dessas atividades $\operatorname{com}(S)=1,0,(I)=0,5,(F)=0$;

- $\quad$ Por fim, tentou-se suprir a possível necessidade de um encontro síncrono utilizando a plataforma Google Meet, sendo feito uma vez por semana em um horário decidido por votação pela turma.

Ao decorrer da aplicação dessa metodologia, em um dos tópicos (Propriedades Geométricas das Seções, foi utilizado o pacote comercial MATLAB® para a formulação de

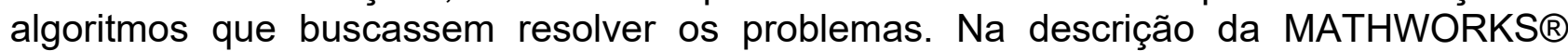
(2020), desenvolvedora do produto, o MATLAB ${ }^{\circledR}$ (MATrix LABoratory) combina um ambiente de trabalho adaptado para análises iterativas e processos de projeto com uma linguagem de programação que expressa a matemática de matrizes e vetores diretamente na tela. Dessa maneira, torna-se mais fácil a programação de soluções que envolvam diretamente operações numéricas se comparada à sua elaboração em linguagens de alto nível tradicionais como $\mathrm{C} / \mathrm{C}++$ ou até mesmo Python. Além disso, o software já é utilizado pela academia na área de Estruturas (área da disciplina em estudo), um exemplo é o de LIMA (2018), que utilizou o software para desenvolver um código baseado no Método dos Elementos Finitos para análise numérica de vigas mistas protendidas. BONWELL E EISON (1991) citam a modelagem de processos e sistemas como um meio de incorporar o ensino ativo na sala de aula. Foi esperado então que sua introdução despertasse o interesse dos alunos tanto pela sua difusão quanto pela sua proposta de otimização de operações as quais seriam repetitivas.

\section{DESENVOLVIMENTO.}

\subsection{Objetivos.}

Este trabalho tem como objetivo verificar se as ferramentas utilizadas (as que foram listadas na introdução) foram úteis para o acompanhamento remoto dos discentes para uso não somente em metodologias remotas e ativas de ensino, mas para tentar estabelecer uma melhor comunicação com o alunado, junto a uma metodologia de aprendizagem que estimule o desenvolvimento individual e a capacidade crítica do discente, facilitando o processo de aprendizado e aperfeiçoamento dos alunos. 


\subsection{Metodologia de análise.}

A metodologia foi proposta à turma 1 da disciplina de Mecânica dos Materiais, ofertada pelo Departamento de Engenharia Estrutural e Construção Civil (DEECC) ao curso de Engenharia Elétrica ( $3^{\circ}$ nível) da Universidade Federal do Ceará (UFC). Com 57 alunos matriculados à princípio, nessa disciplina, foram abordados os seguintes tópicos:

- Introdução à Mecânica dos Materiais - elementos estruturais, classificação dos esforços, apoios e hipóteses simplificadoras da Resistência dos Materiais;

- Estruturas Isostáticas - cálculo de reações e esforços internos (diagramas de momento fletor e esforço cortante) em vigas, e cálculo de reações e esforços internos (método das seções e método dos nós) em treliças;

- Propriedades Geométricas das Seções - Baricentro, Momentos de Primeira Ordem (Momento Estático), Momentos de Segunda Ordem (Momentos de Inércia Retangulares e Polares) e Raio de Giração;

- Tensões - tensão normal e cisalhamentos simples e duplo.

Para a análise da eficácia das ferramentas na facilitação da comunicação, foram feitos questionários no Google Forms para identificar a percepção dos discentes sobre essas ferramentas. A perguntas foram de múltiplas escolhas, para tentar avaliar 0 posicionamento dos alunos acerca das tecnologias, e também dissertativas, para permitir sugestões de algumas possíveis melhorias às atividades.

Já para análise do método proposto de atividades, foi comparado o desempenho dos alunos nas atividades de monitoria ao desempenho nas avaliações do professor, tentando verificar se há alguma relação na média das atividades entregues com a nota final da disciplina.r comunicação com o alunado, junto a uma metodologia de aprendizagem que estimule o desenvolvimento.

\subsection{Resultados e discussão.}

No início da suspenção das atividades presenciais, em meados de março de 2020 , foi proposto o uso do Google Meet para fornecer um momento de interação síncrona com os alunos, no qual poderiam discutir as questões e tirar dúvidas. Essa forma de acompanhamento durou até meados de maio, quando, constatando-se a baixa adesão, os estudantes foram questionados quanto à necessidade dessa atividade síncrona, e se já havia satisfação quanto ao acompanhamento assíncrono. A figura 1 sintetiza os resultados da pesquisa de opinião acerca das atividades síncronas pelo Google Meet. 
Figura 1 - Pequisa de opinião sobre as atividades síncronas.

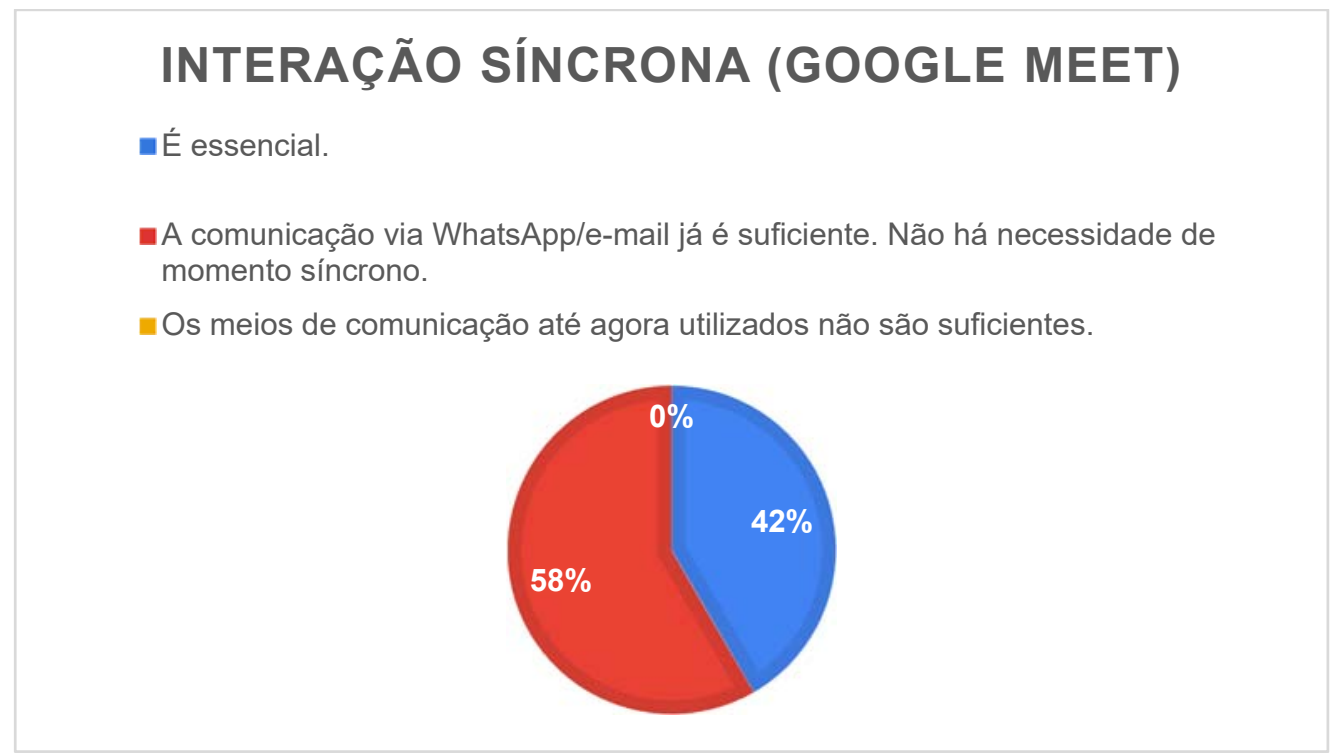

Fonte: $\mathrm{O}$ autor.

A partir dos resultados percebeu-se que a maioria dos estudantes já estava satisfeita com a comunicação assíncrona via WhatsApp e que não havia insatisfação em relação aos meios de comunicação utilizados. Desse momento em diante, atendendo à maioria, optou-se pelo descarte das atividades síncronas e do Google Meet.

Com a consolidação do formato assíncrono, os estudantes foram novamente questionados, ao término da disciplina, quanto à aceitação do uso desse aplicativo para se estabelecer a comunicação com a sala de aula. As respostas foram organizadas na figura 2 , onde 1 representa muito insatisfeito e 5 muito satisfeito.

Figura 2 - Nível de satisfação das interações de monitoria através do aplicativo WhatsApp.

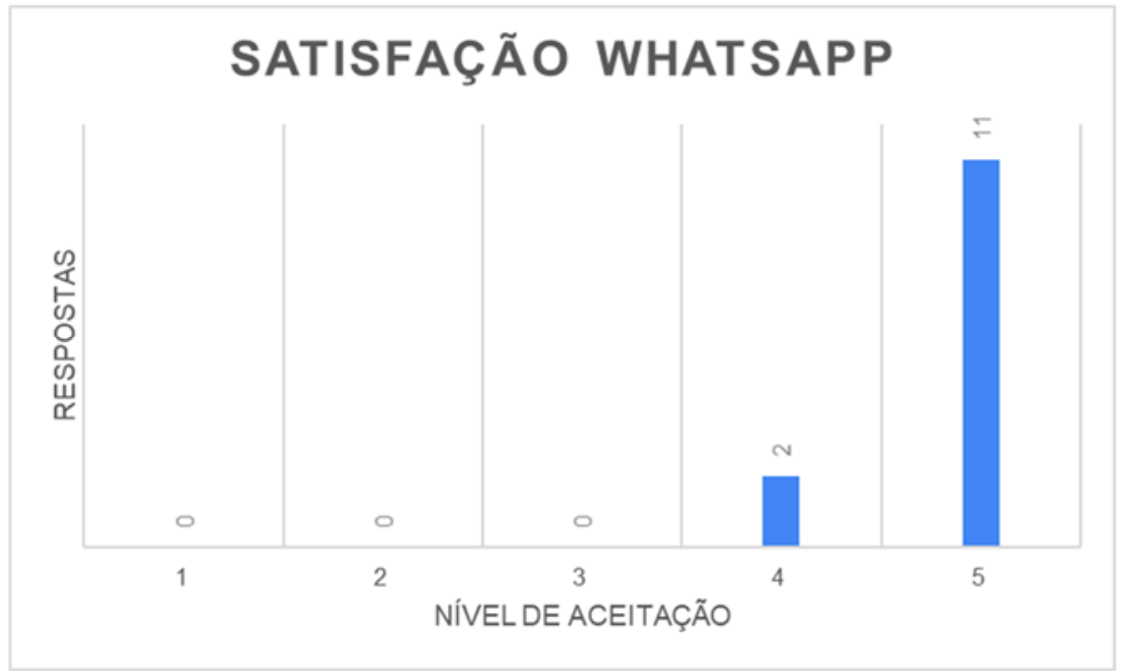

Fonte: $\mathrm{O}$ autor. 
Os estudantes também foram questionados se recomendariam que outros monitores usassem essa ferramenta de comunicação. As opiniões foram sintetizadas na figura 3:

Figura 3 - Nível de recomendação para uso do Aplicativo WhatsApp por outros monitores.

Você recomendaria que outros monitores se comunicassem com a turma por meio desse aplicativo?

13 respostas

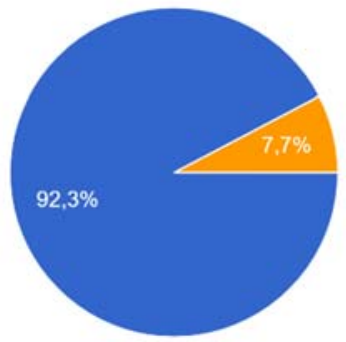

Sim

Indiferente

Năo

Fonte: $\mathrm{O}$ autor.

A forma de organização do material utilizando-se o Google Drive também foi avaliada pelos estudantes. $O$ formato foi aceito como unanimidade pela amostra como retrata a figura 4 , onde 5 representa muito satisfeito.

Figura 4. - Nível de satisfação das interações de monitoria através do google drive.

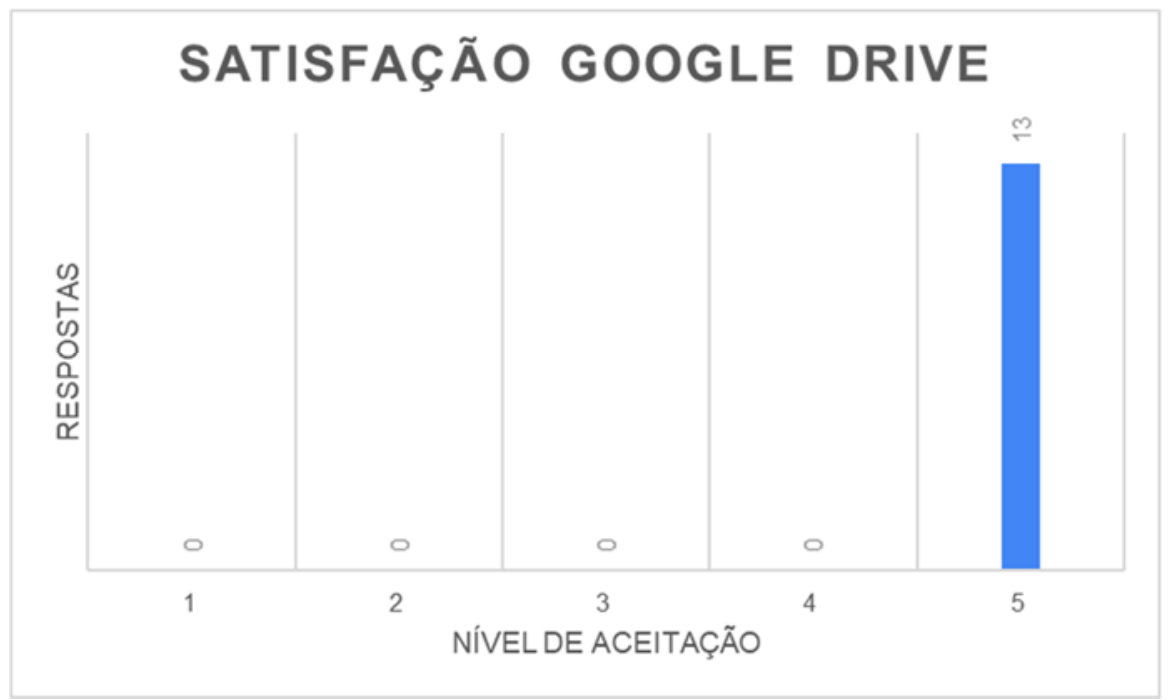

Fonte: $\mathrm{O}$ autor.

A aceitação também foi percebida ao se constatar pela pesquisa de opinião que $100 \%$ dos estudantes que a responderam, recomendariam essa forma de organização a outros monitores. 
Nas perguntas subjetivas, os alunos foram questionados sobre as dificuldades que tiveram na realização das atividades propostas na monitoria. Em resposta, alguns alunos sugeriram que o monitor fizesse pelo menos algumas questões como exemplo e apresentasse à turma antes de lhes atribuir alguma atividade, relatando que apenas os exercícios resolvidos em aula pelo professor ainda não eram o suficiente para o desenvolvimento individual das questões propostas.

Em seguida, foi perguntado sobre o que os alunos gostariam que também fosse disponibilizado na pasta virtual do Google Drive. Então, foi sugerida a gravação de vídeos com a resolução dessas questões, além da resolução autoral (elaborada pelo monitor) em PDF, a qual já era fornecida pelo monitor. Houve também a sugestão de ampliação do uso de algoritmos computacionais aplicados à disciplina, que foram introduzidos na monitoria por meio MATLABß para a resolução de atividades de Propriedades Geométricas das Seções (cálculo de centro de gravidade e momento de inércia).

Por fim, foi verificado o rendimento dos alunos com base na frequência de participação das atividades. Então, dividiu-se a turma em dois grupos: os alunos que obtiveram avaliação "S" em pelo menos $75 \%$ das atividades; e o resto da turma cujo percentual foi abaixo de $75 \%$. Nesses dois grupos, verificou-se o percentual de alunos que conseguiram uma média igual ou superior a 9,0 na disciplina (sem a inclusão da pontuação extra ofertada pelo professor). As figura 5 e 6 mostram respectivamente a relação do desempenho dos alunos com frequência superior a $75 \%$ e os outros colegas.

Figura 5 - Relação Nota nas avaliações vs. Frequência nas monitorias.

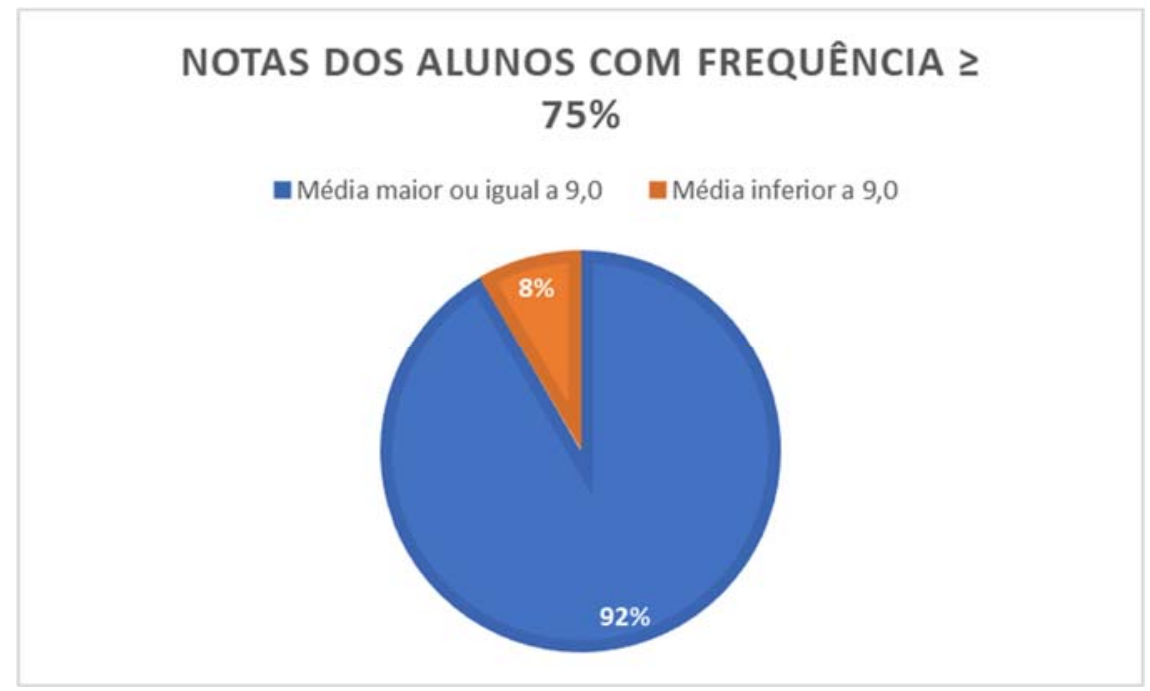

Fonte: $\mathrm{O}$ autor. 
Figura 6 - Relação Nota nas avaliações vs. Frequência nas monitorias.

NOTAS DOS ALUNOS COM FREQUÊNCIA < $75 \%$

- Média maior ou igual a 9,0 = Média inferior a 9,0

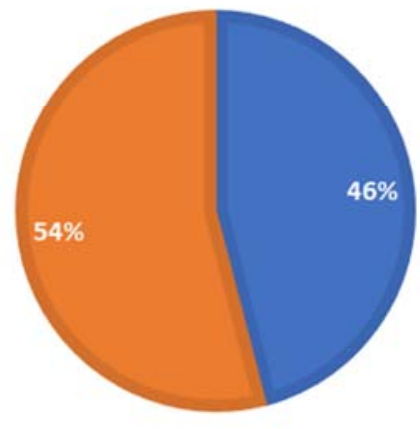

Fonte: $\mathrm{O}$ autor.

Além disso, também foi calculada a média para ambos os grupos, sendo a dos alunos com $75 \%$ ou mais de frequência igual a 9,7 , e a do resto da turma igual 8,3 .

\section{CONCLUSÕES.}

Inicialmente, a partir das pesquisas de opinião e das condições impostas, pôde-se perceber a dificuldade de se trabalhar de maneira síncrona na disciplina. Tanto pelo lado do monitor, pela dependência de equipamentos como mesa digitalizadora para que se possa oferecer um material de qualidade, como pelo lado dos alunos, que dependem de uma boa conexão para acompanhar e que muitas vezes podem não estar disponíveis nos horários das atividades síncronas.

A aceitação do WhatsApp pode ser atribuída não só pela velocidade de comunicação e disseminação do aplicativo na cultura brasileira, mas também por permitir uma informalidade a qual facilita a comunicação, visto que a troca de informações se dá entre alunos, sendo o uso desse aplicativo possivelmente bem mais eficaz do que por e-mail. Uma deficiência clara desse aplicativo em relação a plataformas de sala de aula virtual já consolidadas como o Google Classroom e o Microsoft Teams é a incapacidade de se organizar um material base para a disciplina de maneira organizada. Porém, o uso da pasta compartilhada do Google Drive aparentemente conseguiu suprir essa deficiência, tendo aceitação unânime na amostra. Isso pode ser consequência de que essa associação entre o WhatsApp e o Google Drive forneça rápida comunicação, pelo primeiro, e facilidade de acesso, pelo segundo, pois dessa forma não há necessidade de baixar aplicativos extras ou de se possuir um computador para ser apto a acompanhar as atividades.

Mesmo com aula do professor sendo acompanhada de exemplos, os alunos ainda sentiram dificuldades no desenvolvimento individual das atividades propostas. Uma revisão do assunto pelo monitor, seja por meio de resumos, também acompanhados de exemplos de questão, supõe-se capaz de transpor essa dificuldade da turma. Seguindo as recomendações dos alunos, esse material poderia conter um vídeo-aula acompanhando o documento em PDF, embora que possivelmente haja dificuldade para o monitor produzir os vídeos devido à demanda por equipamentos, tendo em vista as características da disciplina. Há a necessidade de desenvolvimento das soluções passo a passo, sendo 
fundamental o uso da mesa digitalizadora ou equipamento similar que permita ao monitor construir na tela o processo de solução e explicação, de forma similar ao feito no quadro usado em sala de aula presencial

O uso da algoritmos computacionais para a resolução de problemas associados à disciplina foi de certa forma bem visto pelos alunos, tendo em vista as sugestões dos discentes sobre o que poderia ser adicionado à pasta compartilhada da disciplina. De certa forma, essa aceitação se deve à proximidade da aplicação real da solução da Situação Problema proposta e a imposição de raciocínio lógico na programação de máquina contrapondo cálculos repetitivos ao se desenvolver manualmente as operações, fatores os quais deixam a atividade mais interessante ao aluno.

Finalmente, percebeu-se que média final dos alunos com maior participação nas atividades de monitoria foi superior à do restante da turma, tendo um maior percentual no grupo de médias superiores a 9,0. Com os resultados dos cálculos de rendimento nos separados grupos baseados na frequência de participação das atividades, em adição às pesquisas de opinião realizadas, é possível inferir que o material ofertado na monitoria e o formato de metodologia ativa de aplicação dessas atividades contribuíram positivamente para a nota dos alunos e consequentemente para o aumento do conhecimento deles, tendo ainda potencial a ser explorado com base no que foi sugerido

\section{REFERÊNCIAS}

ARAÚJO, U. F., Aprendizagem Baseada em Problemas no Ensino Superior, Summus Editorial, São Paulo, 2009.

BARBOSA, E. F., MOURA, D. C., Metodologias ativas de aprendizagem no ensino de engenharia. XIII International Conference on Engineering and Technology Education, COPEC, Guimarães, Portugal, 2014, p. 110-116.

BONWELL, C., EISON, J. A., Active learning: creating excitement in the classroom, Eric Digests, Publication Identif. ED340272, 1991

CASTRO, E., GONÇALVES, J., BESSA, S., Aplicação da metodologia da problematização. XII Congresso Nacional da Educação (EDUCERE), Formosa, p. 13, 2017.

DIESEL, A., BALDEZ, A.L.S., MARTINS, S.N., Os princípios das metodologias ativas de ensino: uma abordagem teórica. Revista Thema, Lageado, v. 4, $\mathrm{n}^{\circ} 1$, pag. 268 a 288 , 2017.

LIMA, E. M. F. Análise numérica de vigas mistas protendidas com interação parcial. 2018. 96 f. Dissertação (Mestrado em Engenharia Civil)-Centro de Tecnologia, Programa de Pós-Graduação em Engenharia Civil: Estruturas e Construção Civil, Universidade Federal do Ceará, Fortaleza, 2018.

MACEDO, K.D.S., et al. Metodologias ativas de aprendizagem: caminhos possíveis para inovação no ensino em saúde. Escola de Enfermagem Ana Nery, Rio de Janeiro, p. 9, 2018.

Manual MathWorks $®$, MATLAB®. Disponível em:

https://www.mathworks.com/products/matlab.html. Acesso em 19 de novembro de 2020.

MORAN, J., A EAD no Brasil: cenário atual e caminhos viáveis de mudança. Summus Editorial, 2011, p. 45-88.

MORAN, J., A Educação a Distância: pontos e contrapontos, Summus Editorial, 2011, 


\title{
EVALUATION OF DISTANCE COMMUNICATION TOOLS IN TUTORING ACTIVITIES IN ENGINEERING TEACHING.
}

\begin{abstract}
The engineering courses that were taking place in the classroom format, faced a new reality in 2020. With the pandemic of the new Coronavirus taking place, all courses were forced to migrate, on an emergency way, to the educational distance format, thus, the teaching methodology had to be adapted. The use of online communication tools was the first challenge imposed on both students and teachers and the search for a better understanding and efficient use of these tools in the construction of knowledge of engineering students through monitoring activities is the reason for this work. Knowing that in remote education, the interaction between the participants is less intense, the active methodology based in problem-based learning, with assistance of communication tools, was tried in an attempt to keep the student willing to build his knowledge. It was observed that the students showed satisfaction with the fast and informal communication provided by the communication applications. Even with the content being presented by the teacher before the distribution of activities to the students, and with the monitor available at almost any time via the applications, some students still felt difficulties in the individual development of what was proposed. There was a suggestion to produce explanatory videos and a more use of computational algorithms to solve problems. Finally, with the end of the discipline, it was noticed that the grades of the students with the highest participation level were significantly higher than the rest of the class, demonstrating a positive result of the proposed methodology application .
\end{abstract}

Keywords: Educational tutoring activities, Engineering learning, Distance learning, Pandemic 